\title{
BIOLAW AS GLOBAL TOOL FOR HUMAN RIGHTS PROTECTION (PRO HUMAN BIOLAW)
}

\author{
(609979-EPP-1-2019-1-ES-EPPKA2-CBHE-JP) \\ ERASMUS+ CAPACITY BUILDING IN HIGHER EDUCATION \\ CALL EAC/A03/2018 - SELECTION YEAR 2019 \\ pro-human-biolaw@um.es
}
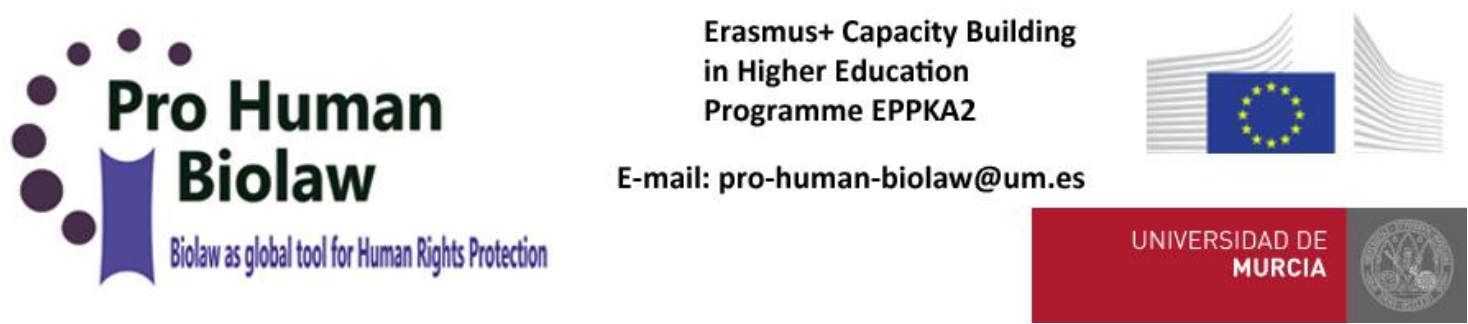

Que el Bioderecho es una disciplina emergente que suscita un creciente interés a nivel nacional e internacional, es una realidad cada vez más incuestionable. La dinámica evolutiva de nuestra sociedad en el ámbito de las biociencias, el progreso investigador y su capacidad de afectar de forma directa al entorno vital sustancial y a la propia entidad constitutiva del ser humano, suscitan interrogantes, dudas éticas y jurídicas y, sobre todo, la necesidad de proporcionar respuestas dinámicas y rigurosas desde el saber jurídico y la reflexión ética.

La necesidad de formar especialistas en esta disciplina se hace cada vez más imperiosa. Las demandas de la sociedad lo son a nivel universal y las nuevas conquistas del progreso científico (como realidad que nace ya con vocación de ser ciencia aplicada), precisan del trabajo interdisciplinar; del concurso de los creadores de las nuevas fronteras de las ciencias experimentales, pero también de la intervención de quienes han de ser garantes del contenido valórico y sustrato ético de los nuevos avances y de quienes han de ser los agentes encargados de trasladar a la sociedad la norma de convivencia que, bajo el prisma de los derechos humanos, permita un desarrollo respetuoso con la persona y su entorno, sostenible y garante de la justicia. 
En el Bioderecho se materializan y convergen esas distintas facetas de la realidad actual que se necesitan entre sí. La respuesta a los retos del mundo moderno, como ya he tenido oportunidad de poner de relieve ante muy distintas instancias y en diferentes escritos, no pueden proceder de una sóla voz; los problemas son ahora mucho más complejos y necesitan de la reflexión conjunta de la ciencia experimental, la ética y el derecho. Se hace imprescindible preguntarse si, frente al creciente poder de intervención de la ciencia en la vida humana, todo lo que es técnicamente posible es éticamente aceptable y, si lo es, dentro de qué límites jurídicos.

Es un hecho incuestionable que, en las últimas décadas, la ciencia ha avanzado a pasos agigantados. Desde finales del siglo XX, el salto exponencial que se ha producido a nivel de investigación y de ejecución práctica de los resultados obtenidos, no es comparable a lo sucedido en tiempos pasados. La magnitud de los resultados derivados de la investigación y la especial incidencia que están teniendo en la entidad constitutiva del ser humano, en su entorno social y ambiental y en el ámbito tecnológico de carácter digital, nos plantea serios dilemas sobre el futuro de la existencia humana, la vida en dignidad y el legado a las generaciones futuras. En la actualidad, la interconexión e interdependencia entre las ciencias es cada vez más evidente y las soluciones justas y respetuosas con la identidad (humana, natural y medioambiental) de nuestro entorno exigen del concurso de un nuevo estilo de trabajo. El bioderecho es esa nueva forma de afrontar la búsqueda de solución a los interrogantes modernos desde planteamientos éticos, con el aval de la ciencia y bajo el marco de en un derecho cercano a la sociedad cuyo referente radica en el imperativo sustentado por los Derechos Humanos.

La Unión Europea y la EACEA (Education, Audiovisual and Culture Executive Agency), han sabido apreciar la trascendencia de estas variables en esta época moderna y ha apostado por el impulso y la financiación de iniciativas como las que se desarrollan en el proyecto Biolaw as global tool for Human Rights protection (Pro Human Biolaw).

Con este proyecto, promovido por la Universidad de Murcia a través del Centro de Estudios en Bioderecho, Ética y Salud (CEBES) y la Escuela Internacional de Doctorado (EIDUM), se pretende aumentar la capacidad de las instituciones asociadas en el ámbito de la enseñanza y la investigación en bioderecho. Proporcionar a los sectores públicos y privados relacionados con la salud, el derecho, el medio ambiente y las nuevas tecnologías (con lo que es a día de hoy la realidad biomédica, biotecnológica y tecnocientífica en general), las herramientas necesarias para ofrecer una respuesta jurídicovalórica a los dilemas biojurídicos y bioéticos actuales. Todo ello bajo la perspectiva de los Derechos Humanos y en atención a los atributos de dignidad que corresponden a toda persona como tal y en relación con su entorno en el momento presente y cara a las generaciones futuras.

El objetivo general del Proyecto Pro Human Biolaw es

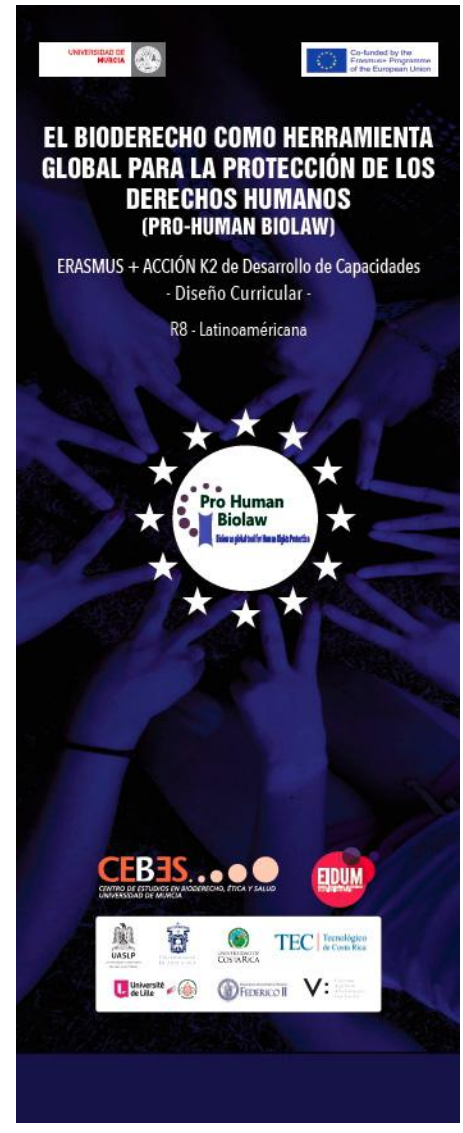
proporcionar a las instituciones las competencias necesarias para profundizar y consolidar la enseñanza e investigación en este campo. Para ello es preciso conformar un elenco de profesorado y de profesionales de alta capacitación en bioderecho y crear de un espacio general de formación, difusión y transferencia en el que combinar la formación especializada en bioderecho, la difusión de la materia, la creación de unos estudios avanzados de calidad y 
excelencia, así como el mantenimiento de una plataforma on-line de enseñanza y de recursos que permita dar cobertura y sustentar en el tiempo esta iniciativa. El proyecto tendrá impacto no solo en las instituciones socias, mejorando su accesibilidad, visibilidad y su capacidad de atracción e influencia, sino también en aquellos sectores que inciden sobre la salud, el entorno ambiental y la tecnología desde la ética, el rigor científico y la reflexión jurídica.

El proyecto está coordinado por la Universidad de Murcia y participan, como universidades socias, otras tres entidades europeas y cuatro latinoamericanas: la Universidad Autónoma San Luis Potosí (México), la Universidad de Guadalajara (México), la Universidad de Costa Rica, el Instituto Tecnológico de Costa Rica, la Universidad de Lille (Francia), la Universidad de la Campania "Luigi Vanvitelli" de Caserta y Nápoles (Italia) y la Universidad Federico II de Nápoles (Italia). Todas estas instituciones, y con la mirada puesta especialmente en el ámbito latinoamericano, son las depositarias del trabajo colaborativo interdisciplinar que incide, principalmente, en el ámbito del derecho en general y en el del derecho sanitario y el de las nuevas tecnologías en particular, la filofofía y la ética aplicada, la biomedicina, la biotecnología, el medio ambiente y la salud y el sustento común que aportan la dignidad de la persona y los derechos humanos.

El consorcio creado es, además, fruto de unas relaciones consolidadas a lo largo de años de trabajo en común. A nivel europeo, con la Universidad francesa de Lille se mantiene una intensa relación en el ámbito de las nuevas tecnologías y bioderecho, hasta el punto de disponer de unos estudios oficiales de Máster que se ofertan de forma conjunta con la Universidad de Murcia. Las Universidades de la Campania y Federico II (Italia) son, desde hace bastantes años, socios destacados a nivel de investigación; el convenio suscrito entre la Universidad de Murcia y el Centro Interuniversitario di Ricerca Bioetica di Napoli (en donde, entre otras, están los Centros Universitarios citados), ha permitido generar investigaciones y resultados conjuntos, así como diversas reuniones científicas en temáticas emergentes de bioderecho. A nivel latinoamericano, con las Universidades mexicanas de Guadalajara y San Luis Potosí, la colaboración a nivel docente e investigador es de larga tradición, no sólo con el intercambio de personal docente y alumnado, sino también a través de convenios específicos en la materia, como el suscrito con el Comité de Ética en la Investigación del Centro Universitario de la Costa de la Universidad de Guadalajara, o el doctorado conjunto en Bioderecho ofertado por la Universidad de Murcia y la de San Luis Potosí, en el que tiene una alta incidencia el tratamiento de los Derechos Humanos. En el ámbito costarricense, junto a los convenios suscritos en materia docente e investigadora tanto con la Universidad de Costa Rica como con el Tecnológico de Costa Rica, se viene llevando a cabo una intensa colaboración en cursos de formación en bioderecho, participación conjunta en reuniones científicas relacionadas con la materia y desarrollo de actividades relacionadas con la temática medio ambiental.

Todo ello nos ha permitido crear un consorcio que surge de una experiencia colaborativa contrastada, en la que el hilo conductor es el desarrollo de las temáticas propias del bioderecho en sus diferentes ámbitos. Salud y biomedicina, nuevas tecnologías, medio ambiente, ética y derechos humanos conforman la existencia de unos equipos interdisciplinares cuyo objetivo es dar respuestas ético-jurídicas a los retos que plantea el avance y descubrimientos de la ciencia moderna; se trata, entre otras cosas, de poner en conexión la experiencia europea con las demandas y eclosión de la temática en los países del entorno latinoamericano. Pro Human Biolaw es una fórmula que va a permitir aproximar los distintos saberes, abrir nuevas vías de trabajo, difundir la realidad actual del bioderecho, estructurar una prospección rigurosa de las necesidades de la sociedad, ofertar una formación que capacite a profesionales y docentes y consolidar una red internacional en bioderecho. 
A través de una serie de objetivos específicos bien determinados, el proyecto se construye mediante:

a) el desarrollo de una oferta académica nueva e innovadora en el ámbito del bioderecho a nivel profesional y en los estudios oficiales en todas sus escalas. Se trata de una oferta que no existe en la mayoría de las instituciones y que debe implantarse como una herramienta básica para capacitar a nuevos profesionales e investigadores. La participación, el trabajo compartido entre distintos equipos académicos y la confección de cursos que integren la perspectiva multidisciplinar y ofrezcan una estructura común de contenidos, son las directrices que han de marcar el camino a seguir, proporcionando contenidos adaptables y capaces de modificarse y ajustarse a medida que surjan nuevos desafíos en el futuro. El proceso que ha de seguirse para definir los contenidos, basado en la prospección y contactos con los sectores de interés, y las sugerencias emanadas de la recopilación e integración de los datos obtenidos, ha de actuar también como un medio de difusión y una forma de ampliar el conocimiento e involucrar a los sectores destinatarios de las temáticas que se abordan.

b) el incremento de la cantidad de recursos humanos de alta capacitación disponibles en los centros de formación y en los sectores profesionales afectados. El reto es llegar a un elevado número de personas y dotar de una capacitación rigurosa implementada con cursos, fondo documental y actividades conexas.

c) el impulso de la enseñanza y el aprendizaje on-line. Es preciso disponer de la tecnología necesaria para poder llegar a docentes, estudiantes y profesionales sin necesidad de la presencialidad que exige la academia tradicional, removiendo así los obstáculos que dificultan el acceso a la educación superior debido a limitaciones profesionales, familiares o de ubicación geográfica. El lanzamiento de una cultura de aprendizaje en red ha de eliminar las barreras para la adquisición de conocimiento y ha de aumentar la innovación, la visibilidad y la capacidad de incursión a nivel nacional e internacional. Es necesario crear una comunidad científica multidisciplinar de académicos y no académicos y que sea lo suficientemente grande como para satisfacer las necesidades de todos los demandantes. Esta es la única forma de garantizar el mantenimiento de las habilidades: investigando, desarrollando la ciencia del bioderecho en sus respectivos ámbitos y transfiriendo resultados a la sociedad en general. Se ha de lograr que, gracias a las nuevas tecnologías para el aprendizaje en red, se preste formación a un gran número de profesionales y estudiantes que permita paliar la escasez de personal especializado y la falta de programas de capacitación dirigidos a dar respuesta a las demandas de asesoramiento y formación existentes. Tanto el ámbito sanitario, como el que incide sobre las nuevas tecnologías, las cuestiones medio ambientales y la salud en general, precisa de personal capacitado para afrontar los desafíos de la biomedicina, la biotecnología y la informática avanzada desde planteamientos éticos, con el aval de la ciencia y bajo el marco de los derechos humanos como garantes de la dignidad y del legado a las generaciones futuras.

d) el mantenimiento de una difusión adecuada de la ciencia del bioderecho. Lo que se pretende es introducir el amplio elenco de temáticas que aborda en el seno del debate social y en los diferentes ámbitos profesionales. Las reuniones científicas y los congresos de divulgación y profundización han de cumplir con su finalidad de aproximar, tanto a la ciudadanía como a los grupos de expertos, las grandes cuestiones que se debaten en este siglo XXI y que están revolucionando el desarrollo y avance tecnocientífico en el ámbito de lo que es el ser humano, sus atributos y los derechos más primarios que le corresponden.

e) La implantación de unos estudios de doctorado en bioderecho de amplio espectro. Con ello se avanzará en una formación de alto nivel en el seno de las sociedades mexicana y 
costarricense en conjunción con la experiencia española y las aportaciones conexas de especialistas franceses e italianos. Un doctorado en bioderecho que aspira a ser un título conjunto a nivel internacional expedido por instituciones de educación superior españolas, mexicanas y costarricenses.

Pro Human Biolaw tiene como finalidad dotar de instrumentos adecuados a especialistas de diferentes ámbitos de procedencia, para permitirles conjugar el saber científico moderno con las exigencias de dignidad que se derivan de los reconocimientos plasmados en los derechos humanos; y ello llevarlo a todos los sectores de la sociedad, prestando especial atención a los ámbitos más vulnerables.

Para el jurista, el dominio del Bioderecho, supone un complemento en su formación de alto valor sobre la base de la realidad social y jurídica de los tiempos modernos. Ante los tribunales de justicia son cada vez más frecuentes las cuestiones de índole biojurídica que exigen un alto grado de conocimiento a nivel científico, ético y jurídico. Para los profesionales de la salud es imprescindible saber los derechos y deberes que les conciernen como tales y los que pertenecen a los pacientes; las responsabilidades que se pueden generar; los protocolos a utilizar y cómo conjugar la realidad valórica con las exigencias de justicia. Para quienes trabajan en el desarrollo de nuevas tecnologías, es primordial conocer el modo de hacerlas compatibles con los derechos a la intimidad y a la privacidad; buscar el lado humano de la tecnología para ponerla al servicio de la persona sin que suponga una intromisión abusiva en su vida. Para quienes se ocupan del medio ambiente y el desarrollo biotecnológico ha de suponer un firme apoyo para unir el ámbito puramente tecnológico a la realidad social y medio ambiental, lo que exige que los resultados respondan a criterios éticos, sean asumibles por la generalidad de la ciudadanía y contribuyan al bienestar de las generaciones futuras.

El Bioderecho nace del método interdisciplinar y del trabajo en equipo. Se sumerge en las ciencias de la vida en sentido omnicomprensivo, que tanto se ocupan de la salud como de las nuevas tecnologías, de la ética medioambiental como de la biotecnología aplicada, del valor de ser persona y de su protección a través de los derechos humanos. Pro Human Biolaw es una apuesta de futuro con el compromiso de construir una sociedad más justa, rescatar la dignidad del ser humano, defender la solidaridad social y jurídica, eliminar los conflictos entre ética y tecnología y crear un derecho que, desde la reflexión valórica, tenga como eje a la persona. Una fórmula para afrontar, desde el trabajo interdisciplinar y la reflexión valórica, los retos que nos propone el mundo moderno. 


\section{$\therefore$ Pro Human \\ Biolaw \\ Biolaw as global tool for Human Rights Protection}

\section{ANEXo \\ Datos del Proyecto}

\begin{tabular}{|c|c|}
\hline \multicolumn{2}{|c|}{$\begin{array}{l}\text { Education, Audiovisual and Culture Executive Agency } \\
\text { Erasmus+: Higher Education - International Capacity Building }\end{array}$} \\
\hline Programme & EPPKA2 - Cooperation for innovation and the exchange of good practices \\
\hline ACtion & CBHE-JP - Capacity Building in higher education - Joint Projects \\
\hline INVOLVED REgIONS & R8 - Latin America \\
\hline APPLICATION COUNTRY & Spain \\
\hline ORGANISATION NAME & University of Murcia (UMU) \\
\hline Partner Country Organisations & $\begin{array}{l}\text { Autonomous University San Luis Potosí (UASLP) - MX } \\
\text { University of Guadalajara (UDG) - MX } \\
\text { University of Costa Rica (UCR) - CR } \\
\text { Technology Institute of Costa Rica (TEC) - CR } \\
\text { University of Lille - FR } \\
\text { University of Campania "Luigi Vanvitelli” - IT } \\
\text { University of Naples Federico II (UNINA) - IT }\end{array}$ \\
\hline Proposal & Call for Proposals 2019 - EAC-A03-2018 \\
\hline Application No & 609979-EPP-1-2019-1-ES-EPPKA2-CBHE-JP \\
\hline TITLE & Pro Human Biolaw - Biolaw as global tool for Human Rights protection \\
\hline MAXIMUM GRANT & $999,489.00 €$ \\
\hline
\end{tabular}

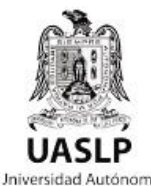

Iniversidad Autónome
de San Luis Potosi

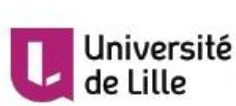

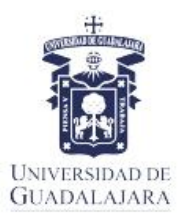

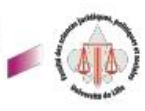

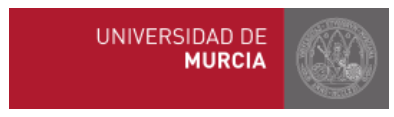

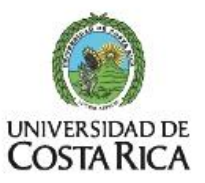

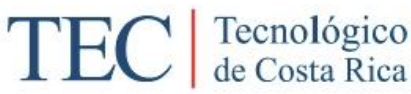
COSTARICA

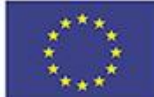

Co-funded by the

Erasmus+ Programme of the European Union
This project has been funded with support from the European Commission. This publication [communication] reflects the views only of the author, and the Commission cannot be held responsible for any use which may be made of the information contained therein. 